\title{
Effects of proarrhythmic drugs on relaxation time and beating pattern in rat engineered heart tissue
}

\author{
Alexandra Eder • Arne Hansen - June Uebeler - Thomas Schulze • \\ Christiane Neuber - Sebastian Schaaf - Lei Yuan - Torsten Christ • \\ Marc A. Vos · Thomas Eschenhagen
}

Received: 7 March 2014/Revised: 27 August 2014/Accepted: 29 August 2014/Published online: 11 September 2014

(C) The Author(s) 2014. This article is published with open access at Springerlink.com

\begin{abstract}
The assessment of proarrhythmic risks of drugs remains challenging. To evaluate the suitability of rat engineered heart tissue (EHT) for detecting proarrhythmic effects. We monitored drug effects on spontaneous contractile activity and, in selected cases, on action potentials (sharp microelectrode) and $\mathrm{Ca}^{2+}$ transients (Fura-2) and contraction under electrical pacing. The $\mathrm{I}_{\mathrm{to}}$-blocker inhibitor 4-aminopyridine increased action potential duration and $\mathrm{T} 2$ and caused aftercontractions, which were abolished by inhibitors of ryanodine receptors (RyR2; JTV-519) or sodium calcium exchanger (NCX; SEA0400). 77 Drugs were then tested at $1-10-100 \times$ free therapeutic plasma
\end{abstract}

A comment to this article is available at doi:10.1007/s00395-0140437-6.

Electronic supplementary material The online version of this article (doi:10.1007/s00395-014-0436-7) contains supplementary material, which is available to authorized users.

A. Eder - A. Hansen - J. Uebeler - T. Schulze - C. Neuber ·

S. Schaaf · T. Christ · T. Eschenhagen ( $\square)$

Department of Experimental Pharmacology and Toxicology,

University Medical Centre Hamburg-Eppendorf, Martinistraße

52, 20246 Hamburg, Germany

e-mail: t.eschenhagen@uke.de

A. Eder - A. Hansen - C. Neuber - S. Schaaf - T. Eschenhagen DZHK (German Centre for Cardiovascular Research), Partner site Hamburg/Kiel/Lübeck, Martinistrasse 52,

20246 Hamburg, Germany

L. Yuan

Danish National Research Foundation Centre for Cardiac

Arrhythmia, Department of Biomedical Sciences, University of

Copenhagen, Copenhagen, Denmark

M. A. Vos

Department of Medical Physiology, UMC Utrecht, Yalelaan 50, 3584 CM Utrecht, The Netherlands concentrations (FTPC): Inhibitors of $\mathrm{I}_{\mathrm{Kr}}, \mathrm{I}_{\mathrm{Ks}}, \mathrm{I}_{\mathrm{to}}$, antiarrhythmics (8), drugs withdrawn from market for torsades des pointes arrhythmias (TdP, 5), drugs with measurable (7) or isolated TdP incidence (13), drugs considered safe (14), 28 new chemical entities (NCE). Inhibitors of $\mathrm{I}_{\mathrm{Kr}}$ or $\mathrm{I}_{\mathrm{Ks}}$ had no effect alone, but substantially prolonged relaxation time (T2) when combined at high concentration. 15/33 drugs associated with TdP and 6/14 drugs considered non-torsadogenic (cibenzoline, diltiazem, ebastine, ketoconazole, moxifloxacin, and phenytoin) induced concentration-dependent $\mathrm{T} 2$ prolongations $(10-100 \times$ FTPC). Bepridil, desipramine, imipramine, thioridazine, and erythromycin induced irregular beating. Three NCE prolonged T2, one reduced force. Drugs inhibiting repolarization prolong relaxation in rat EHTs and cause aftercontractions involving RyR2 and NCX. Insensitivity to $I_{K r}$ inhibitors makes rat EHTs unsuitable as general proarrhythmia screen, but favors detection of effects on $\mathrm{I}_{\mathrm{to}}$, $\mathrm{I}_{\mathrm{Ks}}+\mathrm{I}_{\mathrm{to}}$ or $\mathrm{I}_{\mathrm{Ks}}+\mathrm{I}_{\mathrm{Kr}}$. Screening a large panel of drugs suggests that effects on these currents, in addition to $\mathrm{I}_{\mathrm{Kr}}$, are more common than anticipated.

Keywords Arrhythmia - Torsades des pointes - Drugs . In vitro screening $\cdot$ Engineered heart tissue

\section{Introduction}

Proarrhythmic side effects of drugs can be life-threatening and have led to a number of drug withdrawals from the market [17]. Predicting the arrhythmogenic potential in preclinical drug development is difficult for several reasons [25, 37]. (1) Mechanisms of arrhythmias are complex. This is exemplified by the fact that not only loss-of-function, but also gain-of-function mutations of $\mathrm{K}^{+}$- and $\mathrm{Na}^{+}$-channels 
can cause arrhythmias by promoting ectopic activity, dispersion, and/or re-entry circuits [1]. This corresponds with clinical experiences that both class I $\left(\mathrm{Na}^{+}\right.$-channel blockers) and class III $\left(\mathrm{K}^{+}\right.$-channel blockers) antiarrhythmic drugs have significant proarrhythmic effects [10]. In addition, dysfunction of the sarcoplasmic reticulum $\mathrm{Ca}^{2+}$ release channel (RyR2) or the $\mathrm{SR} \mathrm{Ca}^{2+}$-storage protein calsequestrin underlie catecholaminergic polymorphic ventricular tachycardia, characterized by increased ectopic automaticity in situations of stress. (2) The heart is equipped with several safety mechanisms, explaining why one hit is rarely sufficient to cause symptomatic arrhythmias. Even patients with inherited rhythm disorders experience clinically relevant arrhythmias relatively late in life and/or only under certain trigger situations such as increased sympathetic drive, hypokalemia, drugs, ischemia, or myocardial scars. (3) The existing preclinical test systems have shortcomings [14, 25, 37]. In current routine, new chemical entities (NCE) are tested on cells overexpressing the human eag-related gene (hERG), and those with significant inhibitory activity are excluded from further development. Whereas hERG-tests have documented high sensitivity and specificity for this single ion current and the principal relevance for hERG-inhibition in causing torsades des pointes (TdP) is undisputed, the predictive value of this test is limited [21, 31]. Several drugs have hERG-inhibitory activity without being associated with TdP-arrhythmias (e.g., verapamil). Others have no relevant hERG-activity at clinically used concentrations, but increase the risk of arrhythmias (e.g., mefloquine and phenytoin; [31]).

The Food and Drug Administration and the European Medicines Agency currently recommend an integrated risk assessment, which includes the results of several experimental tests (e.g., hERG, rabbit Purkinje fibers, and dog telemetry) as well as in silico and clinical data [11, 12, 38]. The content of current tests may be improved by characterizing NCEs in a whole panel of cell lines, each expressing a different cloned ion channel. This approach provides a more comprehensive picture of a drugs channelaffecting activity [3, 42], but needs modeling to predict the integrated effect. An alternative strategy is to test drugs directly on cardiac myocytes or more complex cardiac tissues as the "real substrate" for arrhythmias, assuming that any reproducible effect is relevant, independent of its exact mechanism. Unfortunately, isolated adult cardiac myocytes do not beat and, similar to Purkinje fibers or Langendorff-perfused hearts, cannot be examined in large series. Instrumented dogs or rabbits are the most valid models, but cannot be used for screening large number of NCEs, both for ethical and financial reasons.

We have recently developed an automated miniaturized drug screening assay based on our EHT technology and neonatal rat cardiac myocytes, which appears to combine some of the advantages of a relatively intact $3 \mathrm{D}$ cardiac tissue, availability at large numbers, robustness and highcontent readout, particularly analysis of contractile force and kinetics [7, 16, 20]. Experiments with a limited number of model compounds indicated assay sensitivity to detect proarrhythmic effects of drugs [16]. The aim of the present study was to systematically determine the predictive value of the assay by testing a larger number of clinically used compounds with characterized hERG-inhibitory activity and proarrhythmic potential in humans [31] and randomly picked NCEs as well as underlying mechanisms.

\section{Materials and methods}

The investigation conforms to the guide for the care and use of laboratory animals published by the NIH (Publication No. 85-23, revised 1985). A detailed description of methods can be found in the supplemental file.

\section{Cell isolation and EHT-generation}

Heart cells of postnatal d0-d3 Wistar rats and EHTs were prepared as previously described [16, 44]. Fresh neonatal heart cells were mixed with medium, fibrinogen and thrombin, and casted into strip-format $(12 \times 3 \times 3 \mathrm{~mm})$ molds in agarose, in which pairs of elastic silicone posts were placed from above. EHTs maintained for up to 4 weeks.

\section{Measurement of contractile parameters}

Contractile parameters were evaluated as previously described [16]. In principle, the 24-well-plates with EHTs (14-21 days) were put in a gas-, temperature-, and humidity-controlled incubator with glass roof and customized software-controlled video camera placed on top. Contractile parameters of spontaneously beating EHTs were evaluated using an automated figure recognition algorithm. Deflection of the silicone posts was recorded over time and, based on post geometry and elastic modulus of the silicone, used to calculate force, frequency, fractional shortening, contraction and relaxation time (bpm, T1 [from $20 \%$ to peak] and $\mathrm{T} 2$ [from peak to $20 \%$ ], respectively).

Drug screening_video optical analysis

All measurements were performed with 14-21 day old EHTs in fresh serum-free DMEM (Biochrom F04115), supplemented with $10 \mathrm{mM}$ HEPES for $\mathrm{pH}$-steadiness, preincubated at $37{ }^{\circ} \mathrm{C}, 40 \% \mathrm{O}_{2}, 7 \% \mathrm{CO}_{2}, 90 \%$ humidity for $2 \mathrm{~h}$. Measurements were done routinely 1 day after feeding 
with standard EHT medium. The drugs were analyzed in three different concentrations (45 min each, cumulative, $1-100 \times$ free therapeutic plasma concentration [FTPC]). For drug details including solvents see Supplement Table 1. Prior to each measurement, $50 \mathrm{nM}$ epinephrine (Sigma E4643) was added to each well to simulate "physiological" conditions and enhance the likelihood of contractile activity within the $60 \mathrm{~s}$ recording time. Drugs were added under sterile conditions. After $45 \mathrm{~min}$ incubation in a standard incubator, EHTs were transferred to the video optical system (Fig. 1). Due to a sequential mode of measurement (total time $\sim 30 \mathrm{~min}$ ), incubation time varied from the first to the last EHT (45-75 min).

Measurements under perfusion and electrical stimulation, $\mathrm{Ca}^{2+}$-transients, action potentials

Intracellular $\mathrm{Ca}^{2+}$-transients were analyzed in parallel with force under electrical stimulation and continuous perfusion using a novel setup as described previously [35]. The setup consisted of an inverted microscope, a temperature- and $\mathrm{O}_{2} / \mathrm{CO}_{2}$-controlled chamber for the 24-well EHT plate, a flow rate-controlled perfusion system, platinum-iridium wire electrodes for field stimulation, a fluorescence light source (IonOptix Hyperswitch), a photomultiplier, video cameras and software (both IonOptix) for the evaluation of contractile activity (edge detection mode). Experiments were done at $4 \mathrm{ml}$ flow/min (per well) and 2-4 Hz stimulation in modified Tyrode's solution.

For Fura loading, EHTs were incubated in Tyrode's solution containing $10 \mu \mathrm{M}$ Fura2-AM (Invitrogen F1221) and Cremophor EL (0.75\%; Sigma C5135) for $2 \mathrm{~h}$ at $37{ }^{\circ} \mathrm{C}$. The ratio of light emission $(510 \mathrm{~nm})$ at excitation with 340 and $380 \mathrm{~nm}$ light (F340/380 ratio) was used as an index of cytosolic $\mathrm{Ca}^{2+}$-concentration.
Action potentials (APs) were recorded with standard intracellular microelectrodes in intact EHTs. Bath solution contained (in mM): $\mathrm{NaCl} 127, \mathrm{KCl} 4.5, \mathrm{MgCl}_{2} 1.5, \mathrm{CaCl}_{2}$ 1.8, glucose $10, \mathrm{NaHCO}_{3} 22, \mathrm{NaHPO}_{4} 0.42$, equilibrated with $\mathrm{O}_{2}-\mathrm{CO}_{2}$ [95:5] at $36.5 \pm 0.5^{\circ} \mathrm{C}, \mathrm{pH}$ 7.4. Preparations were field-stimulated for at least $1 \mathrm{~h}(2 \mathrm{~Hz})$ before data acquisition. APs were analyzed off-line using the LabChart $^{\circledR}$ software (ADInstruments, Spechbach, Germany).

\section{Statistical analysis}

Data were expressed as mean \pm SEM. Statistical differences were analyzed using the one-way analysis of variance (ANOVA) followed by the Dunett's (all compared to baseline) or Tukey's (all compared to all) adjustment for post hoc multiple comparison, or by paired or unpaired Student's $t$ test, as indicated in the legend of each figure. Results were considered statistically significant if a paired Student's $t$ test revealed a $p$ value of less than 0.05 and the deviation from baseline was at least $15 \%$. This limit was defined after initial series of experiments had shown that formally significant ( $t$ test), but not concentration-dependent effects of drugs often amounted to $\pm 11 \%$. Further support for the $15 \%$ threshold came from quantifying the mean $\pm \mathrm{SD}$ of all baseline measurements ( $n=221$ independent EHTs), which amounted to $99.6 \pm 11.4 \%(\mathrm{SEM} \pm 0.77 \%)$.

\section{Results}

Mechanisms of twitch prolongation and irregular beating pattern

In a previous study with EHTs, we found that the experimental $\mathrm{I}_{\mathrm{Ks}}$-inhibitor chromanol 293b and two drugs known

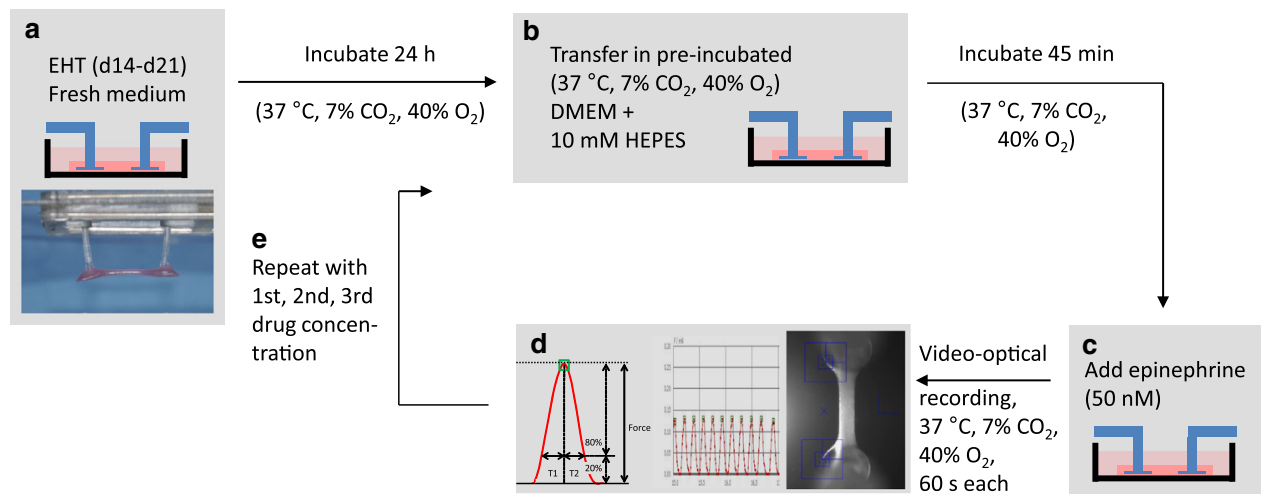

Fig. 1 Schematic illustration of the standard operation procedure for evaluating drug effects. EHTs were subjected to measurements at day 14-21. One day before measurement culture medium was changed. Before evaluation, EHTs were transferred to fresh, preincubated $\left(37{ }^{\circ} \mathrm{C}, 7 \% \mathrm{CO}_{2}, 40 \% \mathrm{O}_{2}\right)$, serum-free DMEM supplemented with $10 \mathrm{mM}$ HEPES for $\mathrm{pH}$ steadiness, and incubated for $45 \mathrm{~min}$.
Epinephrine $(50 \mathrm{nM})$ was added and contractile parameters were analyzed by the video optical system. Thereafter, EHTs were transferred to fresh preincubated DMEM, including HEPES plus the first concentration of a drug, and incubated for $45 \mathrm{~min}$. Epinephrine was added, measurements were done and this circuit started again with the second concentration of the drug 
to inhibit $\mathrm{I}_{\mathrm{Kr}}$ and cause $\mathrm{TdP}$ in humans, quinidine and erythromycin, caused concentration-dependent prolongations of relaxation time T2 [16]. These observations suggested that T2 is a useful surrogate for drug-induced prolongations of repolarization and proarrhythmic effects. To test this hypothesis, we evaluated the effects of the $\mathrm{I}_{\mathrm{to}^{-}}$ inhibitor 4-aminopyridine (4AP; $\mathrm{IC}_{50}$ on $\mathrm{I}_{\mathrm{to}}$ in adult rat ventricular myocytes: $980 \mu \mathrm{M}$ [41]) on EHTs. 4AP prolonged $\mathrm{T} 2$ concentration dependently reaching significance at $3 \mathrm{mM}$ (Fig. 2). At $30 \mathrm{mM}$, EHTs showed extremely prolonged relaxation $(+502 \%)$, comparable to what have been seen previously with chromanol $(+710 \%$; [16]). Prolonged contractions can either be caused by altered intracellular $\mathrm{Ca}^{2+}$ transients or myofilament response to $\mathrm{Ca}^{2+}$. To differentiate between these mechanisms, EHTs were subjected to sharp electrode measurements of action potentials and calcium transients (Fura-2; Fig. 3). Action potential characteristics under basal conditions and electrical stimulation were similar as described previously in this model [16]. 4AP increased action potential duration $\left(\mathrm{APD}_{90}\right)$ from a mean of 113 to $175 \mathrm{~ms}$ (Fig. 3a, b). 4AP also prolonged intracellular $\mathrm{Ca}^{2+}$ transients, whereas the $\beta$ adrenergic agonist epinephrine shortened it, both in the absence and presence of 4AP (Fig. 3c-f). Quinidine, a still prescribed drug for the treatment of atrial fibrillation, also increased $\mathrm{APD}_{90}$ from 179 to $232 \mathrm{~ms}$ at $100 \mu \mathrm{M}$ (Supplemental Fig. 5d). These data supported the interpretation that alterations in T2 reflect similar changes in APD and the kinetics of intracellular $\mathrm{Ca}^{2+}$ transients. Accordingly, a high concentration of caffeine, known to open ryanodine receptors (RyR2) in the sarcoplasmic reticulum (SR), reduced beating rate and induced a large and wide twitch (Supplemental Fig. 1).

4AP not only prolonged $\mathrm{T} 2$, but also induced beat-to-beat irregularities, variations of twitch amplitude and aftercontractions falling into the relaxation phase of prolonged twitches (Fig. 4). To investigate the role of different cellular effector systems in the T2-prolonging effect of repolarization-prolonging compounds, we determined the effect of 4AP in the absence and presence of tetrodotoxin $\left(\mathrm{I}_{\mathrm{Na}}\right)$, tetracaine $\left(\mathrm{I}_{\mathrm{Na}}\right)$, verapamil $\left(\mathrm{I}_{\mathrm{Ca}}\right)$, thapsigargin (SERCA), SEA0400 (sodium calcium exchanger, NCX) or JTV519
Fig. 2 Concentrationdependent effects of the $\mathrm{I}_{\text {to }^{-}}$ blocker 4-aminopyridine (4AP). a-e Representative original recordings of spontaneously beating EHTs in the absence (baseline, only epinephrine $50 \mathrm{nM}$ ) and presence of increasing concentrations of 4AP. $\mathbf{f}$ statistical evaluation of relaxation time (T2; each dot represents one analyzed EHT; $* * p<0.003, * * * p<0.0001$ vs. baseline one-way ANOVA+ Dunnett's post test). The concentration of 4AP was cumulatively increased with 45 min incubation steps per concentration. Note the concentration-dependent prolongation of contraction twitches and the aftercontractions at high concentrations a Baseline

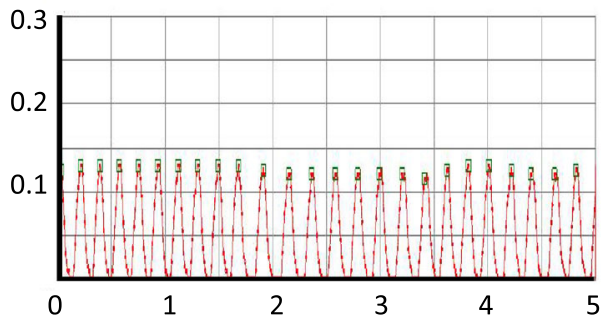

C $4 A P 3 \mathrm{mM}$

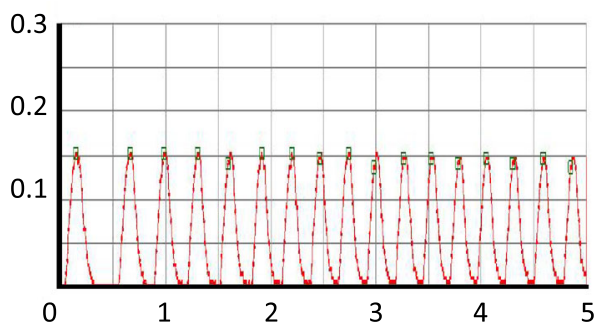

e $\quad 4 A P 30 \mathrm{mM}$

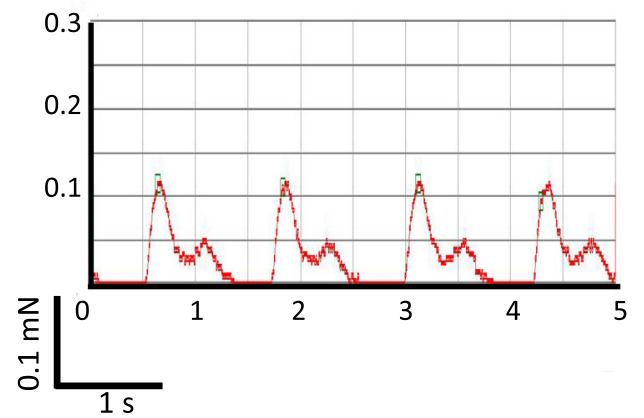

b $4 A P 1 \mathrm{mM}$

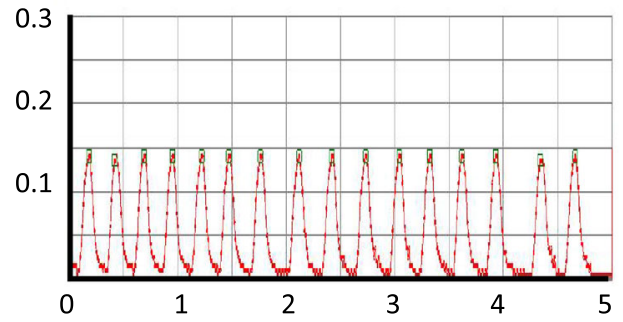

d $4 A P 10 \mathrm{mM}$

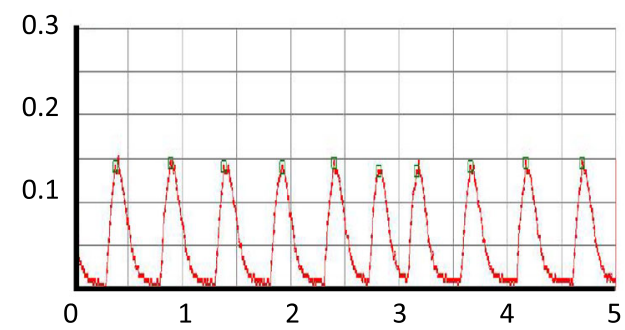

T2

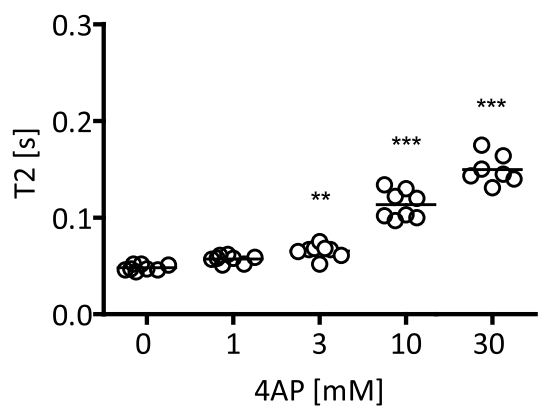




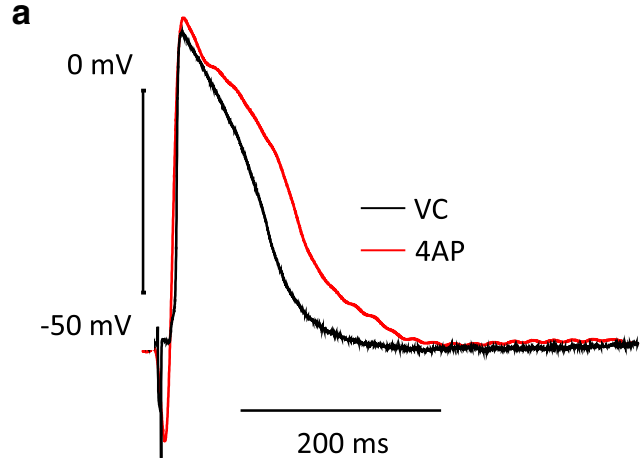

C Vehicle control (VC)
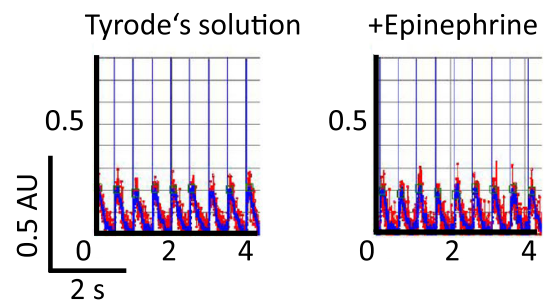

e

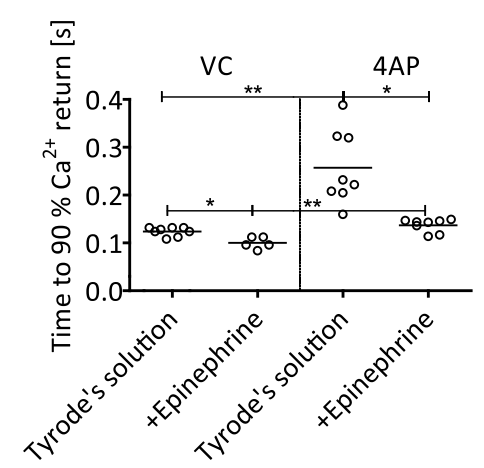

Fig. 3 Effect of 4-aminopyridine (4AP; $10 \mathrm{mM})$, epinephrine $(50 \mathrm{nM})$ or their combination on action potential duration $\left(\mathrm{APD}_{90}\right)$, Fura-2 F340/380 ratio and relaxation time (T2) of electrically stimulated EHTs $(2 \mathrm{~Hz})$. For APD $_{90}$ measurements two independent groups of EHTs $(n=3$, each) were directly perfused with vehicle control (VC) or 4-aminopyridine (4AP), electrically stimulated and contractions recorded. Preparations were stimulated for at least $1 \mathrm{~h}$ before drug exposure and data acquisition. For Fura-2 F340/380 ratio and T2 the two independent groups $(n=8$, each) were preincubated with VC or 4AP. Thereafter, EHTs were perfused and electrically stimulated $(2 \mathrm{~Hz}$; baseline) for $10 \mathrm{~min}$, before epinephrine $(50 \mathrm{nM})$ was added to the perfusion system. a Average peaks for action potentials in the absence (VC, black line) and presence of 4AP (red line). b Statistical evaluation of $\mathrm{APD}_{90}$. c Original recordings of

(RyR2), respectively. Verapamil had no discernible effect, thapsigargin, tetrodotoxin and tetracaine all increased the T2-prolonging effect of 4AP (Supplemental Fig. 2). In contrast, SEA0400 and JTV519 reduced the effect of 4AP (Fig. 4). Both compounds completely abolished beating irregularity and aftercontractions, but only partially T2prolongation. T2 values were 127 (SEA + 4AP) vs. $78 \mathrm{~ms}$ b

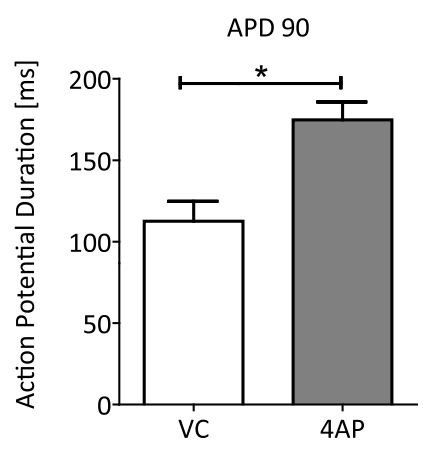

d 4-Aminopyridine (4AP)

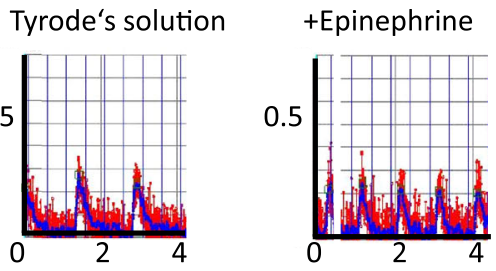

f

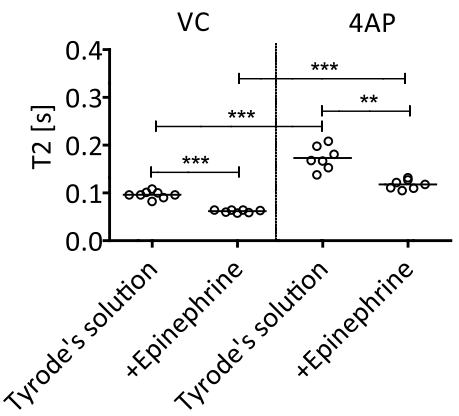

F340/380 ratio transients in the absence (VC, left) and presence of epinephrine (right). d Original recordings in the presence of 4AP alone (left) and 4AP plus epinephrine (right). The red line displays the unfiltered signal, whereas the blue line displays the filtered signal. e Statistical evaluation of time to $90 \%$ return of the F340/380 ratio and F T2. Each point represents one EHT. ${ }^{*} p<0.05$, $* * p<0.003$, ${ }^{* * *} p<0.0001$, Student's $t$ test, paired for comparison within one group (baseline vs. epinephrine) and unpaired for comparison between the two groups (VC vs. 4AP); y-axes for C, D: F340/380 ratio in arbitrary units (AU). Note that $4 \mathrm{AP}$ increased $\mathrm{T} 2$ and time to $90 \%$ return of the F340/380 ratio, both in the absence and presence of epinephrine. Epinephrine alone shortened T2 and F340/380 transients. Note also the failure of capture under 4AP

(SEA alone) and 195 (JTV + 4AP) vs. $80 \mathrm{~ms}$ (JTV alone). This suggests that part of the 4AP-induced T2-prolongation was a direct consequence of action potential prolongation, whereas the SEA- and JTV-sensitive after contractions and pronounced T2-prolongations were mediated by RyR2 and NCX. Similar data were obtained with thioridazine $(30 \mu \mathrm{M}$, data not shown). 
Fig. 4 Effect of inhibitors of the ryanodine-receptor (JTV519 $2 \mu \mathrm{M}$ ) or the sodium calcium exchanger (NCX, SEA0400 $2 \mu \mathrm{M})$ on 4-aminopyridine (4AP $10 \mathrm{mM}$ )-induced prolongations of relaxation and aftercontractions in spontaneously beating EHTs. ad Original recordings of the contractile activity of EHT in the absence of drugs (a, c vehicle control), JTV519 (a, JTV519), SEA0400 (c, SEA0400), 4AP alone (b, d vehicle control) or in the presence of JTV519 (b, JTV5019) or SEA0400 (d, SEA0400). e, $\mathbf{f}$ Time course of the effect of 4AP in the absence (black dots) or presence of JTV519 (e clear squares) or SEA0400 (f clear squares). $n=8$; data are expressed as mean $\pm \mathrm{SEM} ; * p<0.05$, $* * p<0.003, * * * p<0.0001$ vs. vehicle control, one-way ANOVA+ Tukey's post test. Note that both inhibitors prevented the marked 4APinduced $\mathrm{T} 2$ prolongation and after contractions, but did not completely normalize $\mathrm{T} 2$ or beating frequency

\section{a}

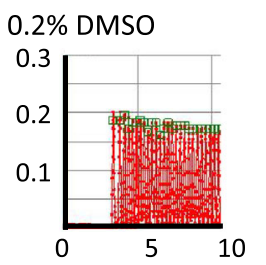

C
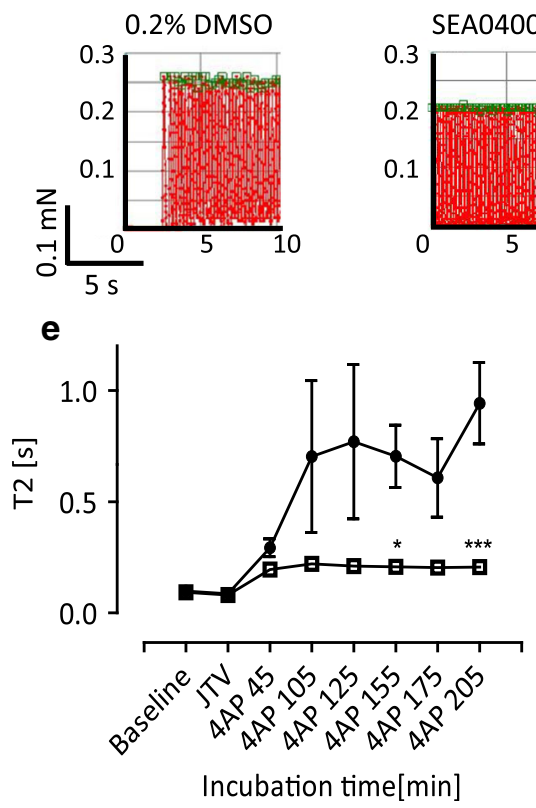

b

4-Aminopyridine
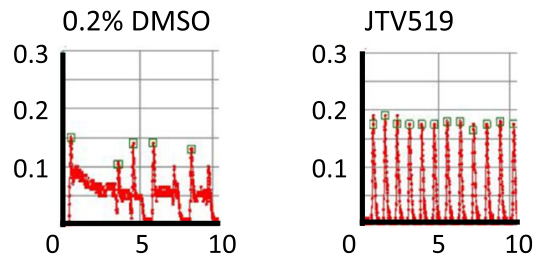

d

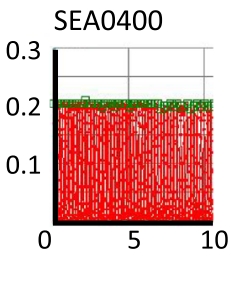

$0.2 \%$ DMSO
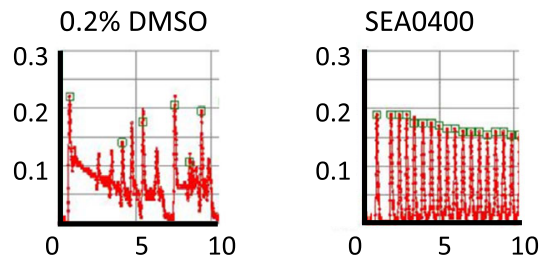

f

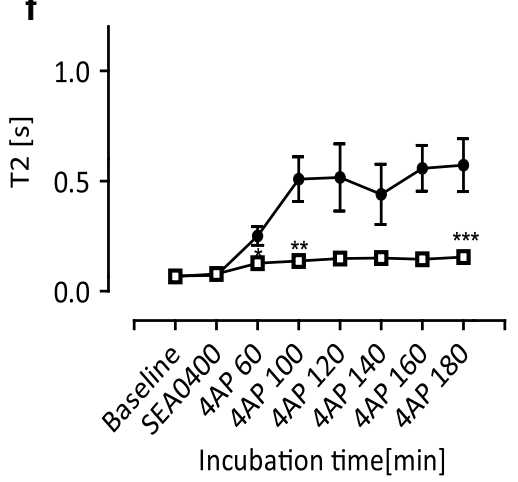

Role of $\mathrm{I}_{\mathrm{Kr}}$ and $\mathrm{I}_{\mathrm{Ks}}$ in $\mathrm{T} 2$ prolongations

The role of $\mathrm{I}_{\mathrm{Kr}}$ and $\mathrm{I}_{\mathrm{Ks}}$ for action potential repolarization in rat heart is still poorly understood [32]. We studied the involvement of these two currents in rat EHTs by applying the reference $\mathrm{I}_{\mathrm{Kr}}$ blocker E-4031 ( $\mathrm{IC}_{50} 7.7 \mathrm{nM}$ [43]) and the selective $\mathrm{I}_{\mathrm{Ks}}$ blocker HMR-1556 ( $\mathrm{IC}_{50} 10.5 \mathrm{nM}$ [36]) alone or in combination (1-1,000 nM, Fig. 5). Neither E-4031 nor HMR-1556 affected T2 even at high concentrations $(1,000 \mathrm{nM})$. The combined application also did not affect T2 at up to $100 \mathrm{nM}$, but caused a substantial increase at $1,000 \mathrm{nM}$ (>8-fold). Given the high selectivity of E-4031 and HMR-1556 for $\mathrm{I}_{\mathrm{Kr}}$ and $\mathrm{I}_{\mathrm{Ks}}$ (HMR-1556 $\mathrm{IC}_{50}$ on $\mathrm{I}_{\mathrm{to}}: 33.9 \mu \mathrm{M}$; on $\mathrm{I}_{\mathrm{Ca}}: 27.5 \mu \mathrm{M}$; on $\mathrm{I}_{\mathrm{Kr}}: 12.6 \mu \mathrm{M}$ [36]), respectively, the effect of the combination suggests a role of these two currents for determining the repolarization reserve in rat EHTs.

Screening of proarrhythmic compounds under spontaneous beating

To test the usefulness of our screening system for the detection of proarrhythmic compounds, we analyzed a large panel of drugs associated with arrhythmias. The selection was made according to a list of drugs published by Redfern and colleagues [31] which related various levels of proarrhythmic risk with inhibition of $\mathrm{I}_{\mathrm{Kr}}$ (hERG). We tested the effect of the 46 compounds of this list which were commercially available (details Supplemental Table 1) at 1-, 10-, and 100-fold FTPC, $n=4-8$ each. In addition, we tested moxifloxacin, an important inhibitor of bacterial gyrases, associated with prolongation of the QTinterval, but not with arrhythmias [28]. The selection of compounds encompassed 8 clinically used antiarrhythmic drugs (Group I), 5 drugs withdrawn from the market for TdP (Group II), 7 drugs with measurable incidence of TdP in humans (Group III), 13 drugs with isolated reports of TdP (Group IV) and 14 drugs devoid of TdP reports and therefore considered safe (Group V; Figs. 6, 7). Under the experimental conditions (which included a $\sim \mathrm{EC}_{50}$ [6] epinephrine concentration, $50 \mathrm{nM}$ ) and in the absence of interventional drugs, EHTs showed a typical and reproducible beating pattern, consisting of periods with high frequency (4.4-4.6 Hz, called "bursts", 8-10 s length) and periods of contractile inactivity (30-40 s; Supplemental Fig. 3). Single twitch kinetics showed a mean \pm SD 

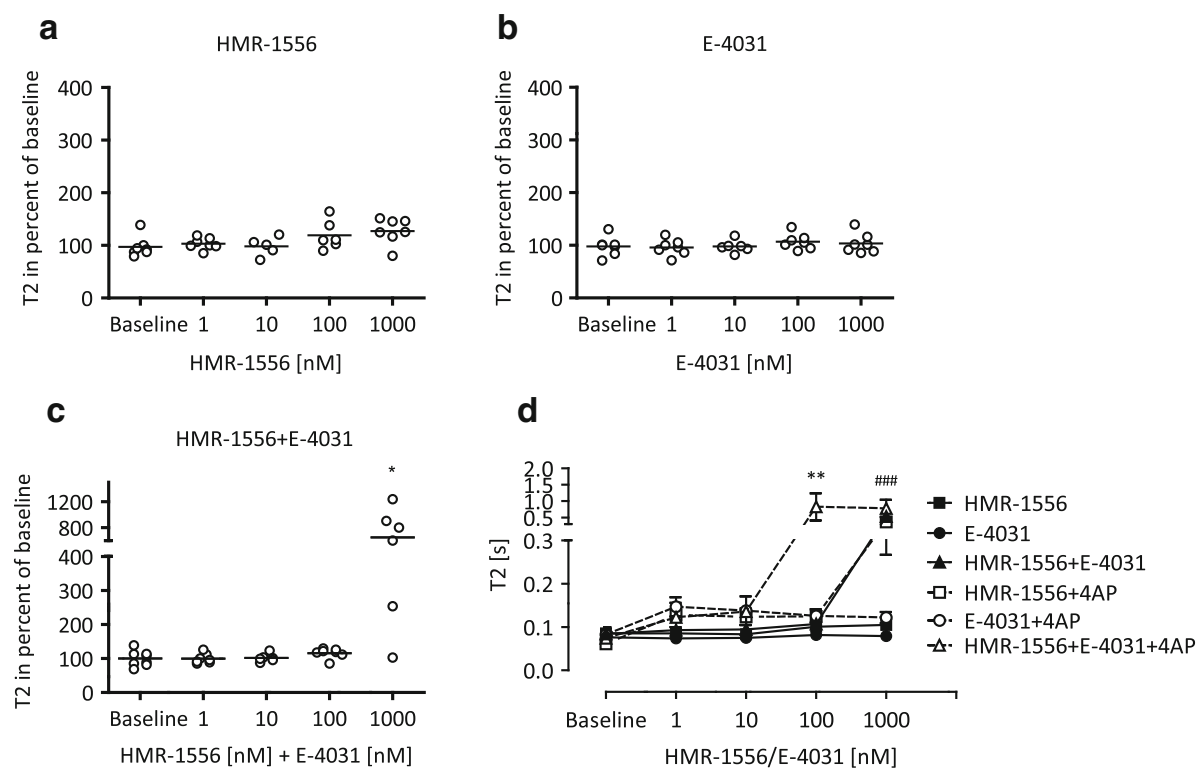

Fig. 5 Role of $I_{K s}$ and $I_{K r}$ in rat EHTs. Statistical evaluation of relaxation time (T2) in the presence of HMR-1556 (a), E-4031(b) or the combination of both (c). Neither HMR-1556 nor E-4031 alone affected T2, but the combination of both led to a marked increase of $\mathrm{T} 2$ at the highest concentration (each $1 \mu \mathrm{M}$ ). d shows the concentration response curves of HMR-1556, E-4031, and the combination of both in the absence (black lines) and presence of 4AP (dashed lines; $3 \mathrm{mM}$ ). In the presence of $4 \mathrm{AP}$, the concentration response

curves for HMR-1556 and HMR-1556 + E-4031 were shifted to the left, whereas the curve for E-4031 alone was not affected. a-c each dot stands for one analyzed EHT; $* p<0.05$ paired Student's $t$ test vs. baseline (50 $\mathrm{nM}$ epinephrine); $\mathbf{d} n=4-8$, data are expressed as mean \pm SEM; $* * p<0.003, * * * p<0.0001$ vs. intervention $+4 \mathrm{AP}$ $\left({ }^{\#}\right.$ HMR-1556; *HMR-1556 + E-4031), one-way ANOVA + Tukey's post test. Note that differences due to the presence of 4AP were not included

(SEM) T1 and T2 of $67 \pm 8( \pm 1)$ and $92 \pm 18( \pm 2) \mathrm{ms}$, respectively. Given that the bursts occurred by chance either fully inside the $60 \mathrm{~s}$ recording window or only partially, the total number of beats per $60 \mathrm{~s}$ was relatively meaningless. In contrast, the frequency of beating in the burst was stable and systematically affected by epinephrine $(+15-20 \%)$ and carbachol (reversed epinephrine effect and induced partial stop; Supplemental Fig. 3).

Given our previous results with erythromycin and quinidine [16], we focused on $\mathrm{T} 1, \mathrm{~T} 2$, and the beating pattern as possible surrogates for arrhythmias. A threshold of $15 \%$ T2 prolongation was considered significant (see Statistics for details). Supplemental Fig. 4 depicts typical examples of drug responses, Figs. 6 and 7 show the T2 effect of 47 drugs according to the Redfern classification. Significant T2 prolongations were observed with $4 / 8$ Redfern Class I drugs (antiarrhythmics; quinidine, disopyramide, sotalol, procainamide; Fig. 6a), 1/5 drugs withdrawn from the market due to TdP (Redfern Class II; cisapride; Fig. 6b), 4/7 drugs with measurable incidence of TdP (Redfern Class III; aprindine, bepridil, flecainide, erythromycin; Fig. 6c), 6/13 drugs with isolated TdP reports (Redfern Class IV; domperidone, sparfloxacin, propafenone, desipramine, fexofenadine, clarithromycin; Fig. 7a), and 6/14 drugs considered safe (ebastine,

ketoconazole, diltiazem, cibenzoline, phenytoin, moxifloxacin; Fig. 7b). Whereas most drugs prolonged T2 only at $100 \times$ FTPC, phenytoin exerted this effect already at $1 \times$ FTPC. In addition, five compounds (bepridil, desipramine, imipramine, thioridazine, and erythromycin) induced irregular beating as the primary effect, characterized by a "sinusoidal" fluctuation of twitch amplitudes (Supplemental Fig. 4a). The effect was time dependent and was followed by complete stop of beating. It occurred at concentrations of 30-100 fold FTPC, i.e., 3-10 ( -600 in case of thioridazine) fold $\mathrm{hERG} \mathrm{IC}_{50}$. Bepridil, desipramine, and erythromycin increased $\mathrm{T} 2$ in addition to inducing beating irregularity.

In aggregate, the rat EHT responses categorized the 47 drugs in 4 groups (Fig. 8). Group 1 caused irregular beating as the main effect $(n=5)$, group 2 induced a concentration-dependent increase in T2 $(n=18)$. T2effective concentrations were 5 fold (domperidone, disopyramide) to 100 fold FTPC (e.g., ebastine, diltiazem, sotalol, or moxifloxacin). Group $3(n=7)$ had variable effects on EHT contraction including prolongation of contraction time T1 (haloperidol, sertindole, diphenhydramine, and mefloquine), shortening of $\mathrm{T} 2$ (terfenadine) and a decrease in force (verapamil and astemizole). The negative inotropic effect of verapamil 

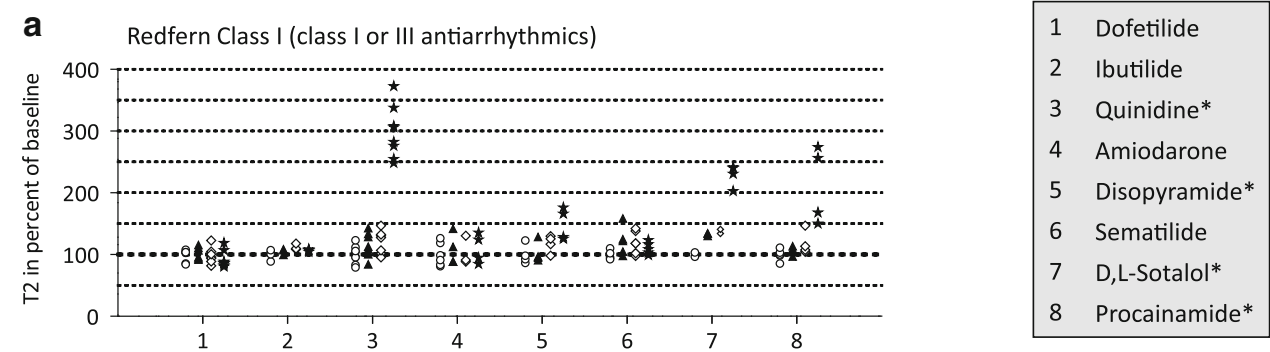
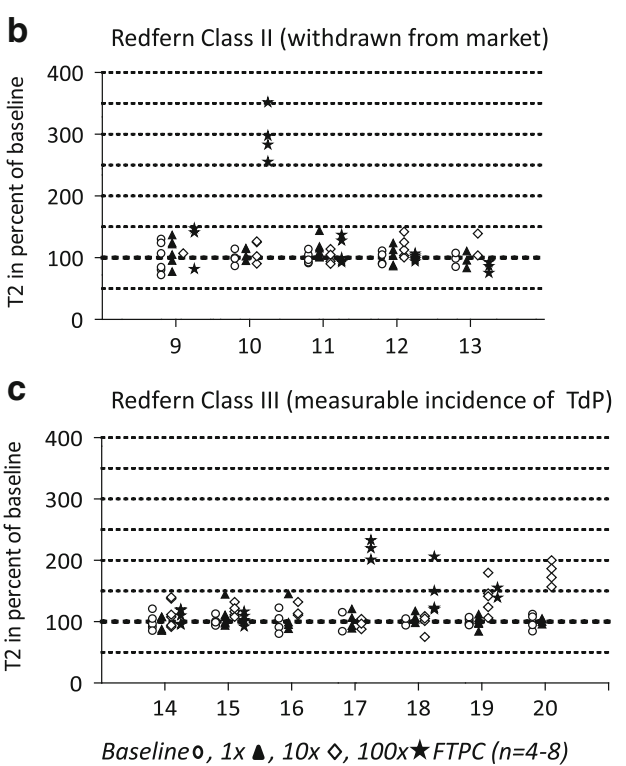

Fig. 6 Effects of class I-III (Redfern) drugs on rat EHT relaxation time (T2). a Shows the effect of antiarrhythmics (class I), b of drugs which were withdrawn from the market (class II) and $\mathbf{c}$ of drugs with measureable incidence of TdP. Each symbol reflects one analyzed EHT. Different concentrations are indicated by different symbols (baseline $0,1 \times \Lambda, 10 \times \diamond, 100 \times$ FTPC). Statistically significant

was already seen at $300 \mathrm{nM}$, approximately 4-fold FTPC. Group 4 encompassed 17 compounds without any effect on the parameters studied. It contained specific $\mathrm{I}_{\mathrm{Kr}}$-blockers such as ibutilide and dofetilide, which was in contrast to the results obtained with human EHTs [33].

Effects of drugs under electrical pacing

Drugs which caused prolongations of $\mathrm{T} 2$ also reduced beating rate in the bursts in many cases (Supplemental Fig. 4), raising the question which effect is the cause and which the consequence. We therefore measured EHT contractility under continuous electrical stimulation and perfusion and carefully evaluated the time course of the effects of quinidine $(100 \mu \mathrm{M})$ and erythromycin $(1 \mathrm{mM}$; Supplemental Fig. 5). Quinidine first induced a marked, changes in $\mathrm{T} 2$ are indicated by an asterisk behind the drug name. Effects other than T2 prolongations are indicated by hashtag (e.g., thioridazine: irregular beating). Data are expressed as percent of baseline ( $50 \mathrm{nM}$ epinephrine). ${ }^{*} p<0.05$ paired Student's $t$ test vs. baseline and at least $15 \%$ difference between means

time-dependent prolongation of $\mathrm{T} 2$ and a reduction in force, which was then followed by the development of a slower rhythm independent of pacing. Erythromycin also first prolonged $\mathrm{T} 2$, reduced force and then induced an autonomous chaotic contraction pattern. Effects of both drugs were fully reversible after washout. The data suggest that the drugs exert primary effects on processes underlying relaxation and that slowing of rate is a consequence.

Screening of new chemical entities (NCEs) under spontaneous beating

To get a rough estimate of the frequency of rat EHT effects in a non-selected group of drugs a chemical library was analyzed. NCEs were purchased from Maybridge and randomly chosen by respecting the rule of five [26]. 28 
Fig. 7 Effects of class IV-V (Redfern) drugs on rat EHT relaxation time (T2). a Shows the effect of drugs with isolated reports of TdP (class IV) and b with no reports of TdP in humans (class V). Each symbol reflects one analyzed EHT. Different concentrations are indicated by different symbols (baseline $0,1 \times \Lambda, 10 \times \diamond$, $100 \times$ FTPC). Statistical significant changes in $\mathrm{T} 2$ are indicated by an asterisk behind the drug name. Effects other than $\mathrm{T} 2$ prolongations are indicated by hashtag (e.g., verapamil: negative inotropic effect). Data are expressed as percent of baseline $(50 \mathrm{nM}$ epinephrine). ${ }^{*} p<0.05$ paired Student's $t$ test vs. baseline and at least $15 \%$ difference between means
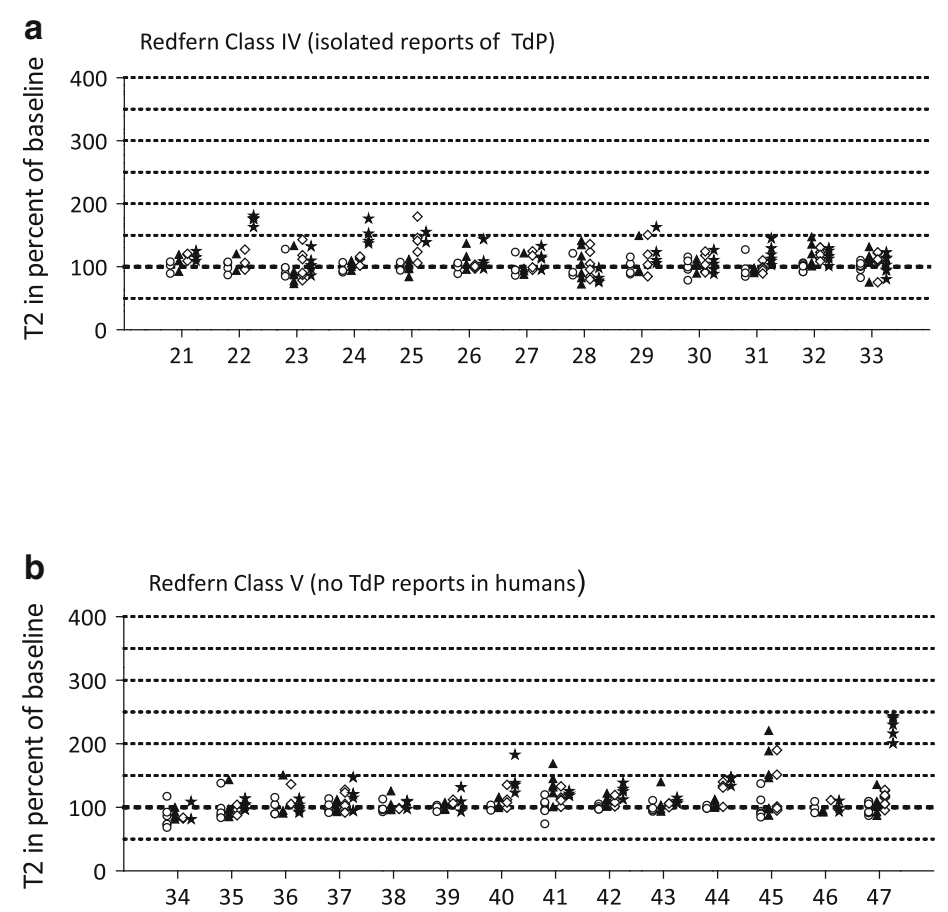

Baseline o, $1 x \star, 10 x \diamond, 100 x \star F T P C(n=4-8)$

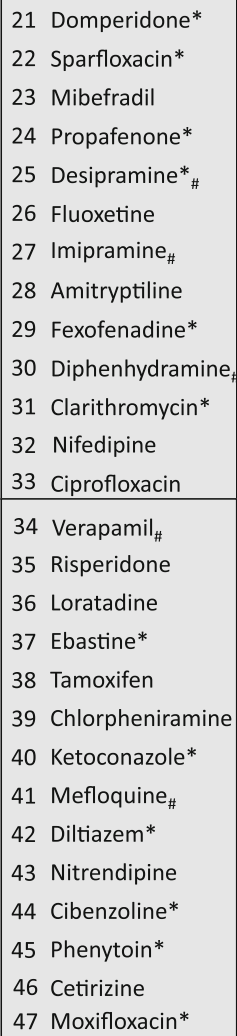

NCEs were tested at $0.1,1$, and $10 \mu \mathrm{M}$. Most NCEs had no effect on EHT contractility. Three NCEs prolonged T2 and one reduced force. In two additional cases EHTs stopped beating and didn't react to electrical pacing (Supplemental Table 2). Overall, an effect on EHT contractility was observed in $21 \%$ of the investigated NCEs.

\section{Discussion}

Assessing the proarrhythmic risks of drugs remains a challenge in preclinical drug development. Current experimental models in preclinical toxicology determine the effect of NCEs on ion channels commonly involved in arrhythmogenic drug effects, particularly hERG channels [39], on electrophysiological surrogates of arrhythmias such as triangulation in Langendorff-perfused rabbit hearts $[23,24]$, on action potential duration in rabbit Purkinje fibers [15] or the QTc intervall in telemetrically surveyed dogs [13]. Newer models with higher throughput and/or a human cardiomyocyte context include measurements of heart rate in zebrafish [27], of $\mathrm{Ca}^{2+}$ transients in isolated guinea-pig [30] or human pluripotent stem cell (hPSC)derived cardiomyocytes [5] or of electric field potentials in hPSC-cardiomyocytes [2]. The present study in rat EHTs is, to the best of our knowledge, the largest head-to-head comparison of proarrhythmic drugs performed so far. It showed that time of relaxation (T2) in rat EHTs is essentially insensitive to selective blockers of $\mathrm{I}_{\mathrm{Kr}}$ or $\mathrm{I}_{\mathrm{Ks}}$, but prolonged by inhibition of $\mathrm{I}_{\text {to }}$, combined full inhibition of $\mathrm{I}_{\mathrm{Kr}}$ and $\mathrm{I}_{\mathrm{Ks}}$ or combined inhibition of $\mathrm{I}_{\mathrm{Ks}}$ and $\mathrm{I}_{\mathrm{to}}$. This suggests that rat EHTs monitor mainly $\mathrm{I}_{\mathrm{to}}$ and $\mathrm{I}_{\mathrm{Ks}}$ effects of drugs. The $\mathrm{I}_{\mathrm{to}_{\mathrm{o}}}$-blocker $4 \mathrm{AP}$ also prolonged action potential duration and $\mathrm{Ca}^{2+}$ transients at T2-effective concentrations, indicating that, in this experimental model, T2 prolongation follow similar prolongations of repolarization and $\mathrm{Ca}^{2+}$ transients. The data suggest that, for drug screening purposes, $\mathrm{T} 2$ is a useful surrogate for time of repolarization in rat EHTs. The high percentage of known proarrhythmic drugs that induced concentration-dependent T2 prolongations, aftercontractions and/or irregular beating (group 1 and 2,23/38 =61\%) indicates that many clinically used drugs possess effects on $\mathrm{I}_{\text {to }}$ and/or $\mathrm{I}_{\mathrm{Ks}}$ in addition to their well-characterized inhibitory action of $\mathrm{I}_{\mathrm{Kr}}(\mathrm{hERG})$.

Characterization of rat EHTs as a test system for testing proarrhythmic drugs

The underlying hypothesis of this study was that repolarization-inhibiting drugs prolong action potential duration 


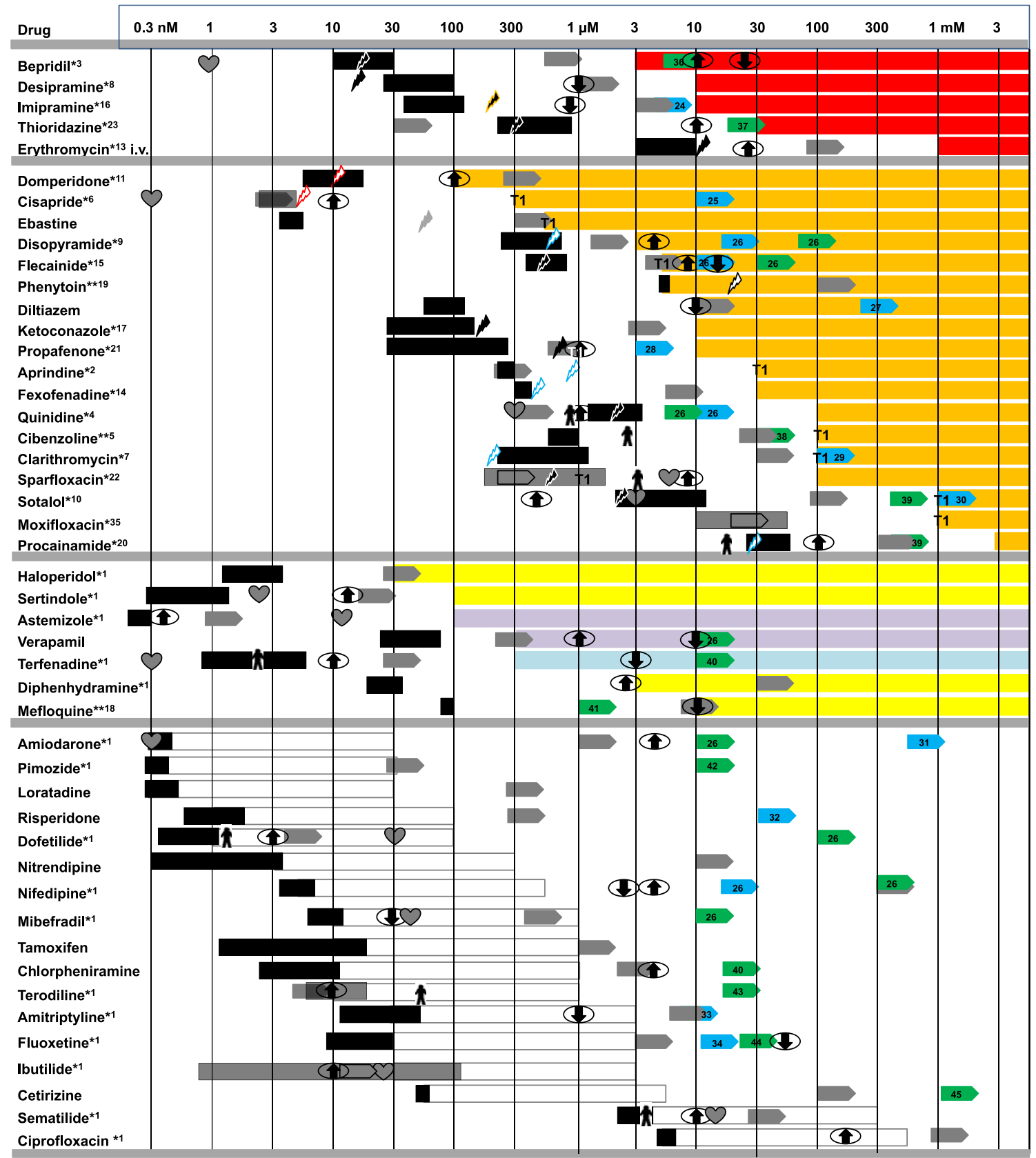

Fig. 8 Grouping of drugs according to the type and the concentration-dependence of the effects on EHTs. All drugs were tested in $1 \times$, $10 \times$, and $100 \times$ free therapeutic plasma concentration (FTPC) in the presence of $50 \mathrm{nM}$ epinephrine. The absolute concentration range is indicated in the first row. Group 1 encompasses drugs (red) that induced polymorphic arrhythmias, group 2 (orange) prolongations of relaxation (T2), group 3 prolongations of contraction (T1, yellow), shortening of T2 (blue), or negative inotropic effects (violet). Group 4 (white) drugs exerted no significant effect. The colored bars in group 1-3 range from the lowest tested concentration in which the indicated effect occurred (generally $10-100 \times$ FTPC) to $3 \mathrm{mM}$, regardless of whether this concentration has been tested. The white bars in group 4 indicate the tested concentration range. Also, indicated are $h E R G / I_{K r}$ lowest published $\mathrm{IC}_{50}$ upward $\longrightarrow$; lowest published $\mathrm{IC}_{50}$ values on $\mathrm{I}_{\mathrm{to}}$ upward $\quad$; lowest published $\mathrm{IC}_{50}$ values on $\mathrm{I}_{\mathrm{Ks}}$ upward concentrations associated with $10-20 \%$ increases in $\mathrm{APD}_{90}(\mathbf{D}$ with decreases in $\mathrm{APD}_{90}(\mathrm{D}$; QTc-prolongation in vivo $\nabla$; increased QTc in humans; estimated plasma concentration (ep) for $\mathrm{TdP}$ case reports in humans $\mathrm{d}$; ep for TdP case reports in combination with other drugs/diseases ; ep for TdP case reports in combination with a cytochrom P450 inhibitor ep for TdP case reports in children ; ep QTc case reports ; asterisk TdP in humans known; double asterisk other types of arrhythmias in humans known. The type of depiction and most information independent of EHTs were adopted from Redfern et al. (2003). Case reports (indicated by asterisk), estimated plasma concentration and $\mathrm{IC}_{50}$ values on $\mathrm{I}_{\mathrm{to}} / \mathrm{I}_{\mathrm{Ks}}$ were obtained by a literature search 
and $\mathrm{Ca}^{2+}$ influx and thereby time of relaxation, a parameter easily assessable in EHT. EHTs are engineered threedimensional cardiac tissue constructs in which cardiac cells are embedded in a fibrin matrix and, over 10-14 days, form a highly organized longitudinally oriented network [16]. The 24-well mini-EHTs system is designed for a robust, automated measurement of spontaneous or electrically stimulated contractile activity at a medium throughput scale. It is stable for weeks and has the advantage that measurements can be done at near-physiological conditions: $37{ }^{\circ} \mathrm{C}$, steady state beating (not dying preparations such as isolated myocytes, papillary muscles, Purkinje fibers, and Langendorff hearts), auxotonically work-performing heart muscle constructs, and absence of invasive experimental interventions (e.g., patch clamp or microelectrodes). On the other hand, it is not well suited for measuring effects of drugs on action potentials, field potentials, or ion channels directly. We therefore performed several experiments to evaluate whether the relaxation time $\mathrm{T} 2$ is indeed a suitable surrogate of time of repolarization.

The following observations favor this assumption. (1) $\mathrm{T} 2$ is easily determined and highly reproducible (11\% SD, $0.77 \%$ SEM, $n=221$ ). (2) The duration of contraction and relaxation corresponds well with that of action potentials in rat EHTs and both were prolonged in the presence of $4 \mathrm{AP}$ as well as quinidine. Action potential duration $\left(\mathrm{APD}_{90}\right)$ measured with sharp microelectrodes at $36{ }^{\circ} \mathrm{C}$ amounted to a mean of $113 \mathrm{~ms}$, time to $90 \% \mathrm{Ca}^{2+}$ return to a mean of $124 \mathrm{~ms}$ at $37{ }^{\circ} \mathrm{C}$, and $\mathrm{T} 1+\mathrm{T} 2$ (at $80 \%$ relaxation) amounted to a mean of $159 \mathrm{~ms}$ at $37{ }^{\circ} \mathrm{C}$ (this study and [16]). Thus, it is reasonable to assume that prolongations of repolarization affect T2. (3) Drugs with well-characterized actions on $\mathrm{I}_{\mathrm{to}}$ [41], $\mathrm{I}_{\mathrm{Kr}}$ [43], and $\mathrm{I}_{\mathrm{Ks}}$ [36] had reproducible, concentration-dependent and reversible effects on T2. 4AP started to prolong T2 at $3 \mathrm{mM}$, which corresponds to 3 -fold $\mathrm{IC}_{50}$ in adult rat cardiac myocytes [41] and confirms the prominent role of $\mathrm{I}_{\text {to }}$ for rodent heart repolarization [19]. The lack of effect of E-4031, dofetilide, ibutilide, and HMR-1556 even at 100 -fold $\mathrm{IC}_{50}$ concentrations confirms the minor role of $\mathrm{I}_{\mathrm{Kr}}$ and $\mathrm{I}_{\mathrm{Ks}}$ for repolarization in rat cardiac myocytes [29]. Interestingly, however, the combined application of high concentrations of E-4031 and HMR-1556 markedly prolonged T2. Given the high selectivity of both drugs for $\mathrm{I}_{\mathrm{Kr}}$ and $\mathrm{I}_{\mathrm{Ks}}[36,41]$, respectively, the data suggest that $\mathrm{I}_{\mathrm{Kr}}$ and $\mathrm{I}_{\mathrm{Ks}}$ can substitute for each other, but together play a role for repolarization in rat EHTs. The strong effect of HMR-1556 in the presence of $3 \mathrm{mM} 4 \mathrm{AP}$ (and the lack of an E-4031 effect under this condition) suggests a greater role for $\mathrm{I}_{\mathrm{Ks}}$ than $\mathrm{I}_{\mathrm{Kr}}$. The necessary channel subunits are expressed in rat EHTs (KCNH2a, KCNQ1a, and KCNE1/E2 mRNAs are present in rat EHTs [16]).
Whereas all this argues for repolarization as an important parameter of relaxation time $\mathrm{T} 2$ in our system, it is obvious that other mechanisms have to be considered and that $\mathrm{T} 2$ prolongations alone do not prove effects on repolarization. Any effect on beating rate will affect $\mathrm{T} 2$ because of the well-known frequency-dependent acceleration of relaxation. Such effect could be excluded in case of quinidine and erythromycin (Supplemental Fig. 5), but may play a role in other cases. Drugs that directly affect intracellular $\mathrm{Ca}^{2+}$ handling could alter $\mathrm{T} 2$ independently of repolarization. Indeed, caffeine at a high concentration $(5 \mathrm{mM})$ which enhances the open probability of RyR2 reduced beating rate and caused widening of contraction twitches (Supplemental Fig. 1). Thapsigargin, a selective inhibitor of SERCA, did not affect time of relaxation as such $(3-30 \mathrm{nM})$, but aggravated the relaxation-slowing effect of 4AP (Supplemental Fig. 2). Another possibility to affect T2 independently of repolarization is an effect on myofilament $\mathrm{Ca}^{2+}$ sensitivity. $\mathrm{Ca}^{2+}$ sensitizers such as EMD 57033 [34] shift the force-pCa curve to the left and thereby prolong relaxation as recently shown in mouse EHTs [35]. The unexpected T2-prolongation under diltiazem may represent this mechanism. A study in isolated adult rat ventricular myocytes reported a mild myofilament $\mathrm{Ca}^{2+}$ sensitizing effect of diltiazem [9]. Such an effect may also explain the unexpected lack of significant negative inotropic effects of this compound at the highest tested concentration $(10 \mu \mathrm{M})$ which was in contrast to verapamil. Conversely, epinephrine (Supplemental Fig. 3) and all other cAMP-dependent drugs increase the PKA-dependent phosphorylation of troponin I, myosin binding protein $\mathrm{C}$ and others and shift the curve to the right. This favors relaxation and abbreviates T2. Taken together, T2-prolongation of rat EHTs can be caused not only by blockers of repolarization (mainly $\mathrm{I}_{\mathrm{to}}, \mathrm{I}_{\mathrm{Ks}}$, very little contribution of $\mathrm{I}_{\mathrm{Kr}}$ ), but also rate-slowing drugs, $\mathrm{Ca}^{2+}$ sensitizers or blockers of cAMP-dependent pathways such as carbachol (Supplemental Fig. 3), which needs to be considered in drug screening efforts.

The pronounced prolongation of relaxation at high concentrations of $4 \mathrm{AP}$ and aftercontractions were sensitive to both SEA0400 and JTV519 (Fig. 4), but relaxation remained significantly prolonged in their presence ( $\sim 200 \%$ ). This finding is interesting as it suggests that the primary effect of $4 \mathrm{AP}$, the inhibition of repolarization, has time-dependent (see time course in Fig. 4) secondary effects that affect time of relaxation. Very likely, this secondary effect corresponds to increased filling of the SR with $\mathrm{Ca}^{2+}$ with the final consequence of RyR2-mediated spontaneous $\mathrm{Ca}^{2+}$ release and NCX-mediated $\mathrm{Ca}^{2+}$ extrusion. The latter transport is electrogenic and contributes to prolonged depolarization and after depolarizations, visible in our system as marked prolongations of $\mathrm{T} 2$ and 
aftercontractions. An interesting speculation is that group 1 (Fig. 8) drugs directly interfere with RyR2 and/or NCX or SR function and therefore induce irregular beating, whereas group 2 drugs primarily affect repolarization and therefore prolong $\mathrm{T} 2$ before causing extra beats. On the other side, group 1 and 2 effects cannot be firmly separated because three group 1 drugs also caused T2 prolongations. Interestingly, tetracaine (also flecainide $(0.5 \mu \mathrm{M})$; data not shown) did not mimic the effect of JTV519 (Supplemental Fig. 2), although both have inhibitory effects on RyR2mediated $\mathrm{Ca}^{2+}$ release $[18,40]$. Possibly, their main effect on $\mathrm{I}_{\mathrm{Na}}$ which is not shared by JTV519 could explain the difference.

Drug-induced relaxation slowing and beating irregularities in rat EHTs

We selected drugs according to a list published by Redfern and colleagues [31] that grouped drugs according to their proarrhythmic potential. 15/33 drugs with various degree of risk for TdP (Class I-IV) showed T2 prolongation or beating irregularities in our system (group 1 and 2). The percentage did not clearly differ between those that were withdrawn from the market for $\operatorname{TdP}(1 / 5)$ and those with only isolated TdP reports (6/13). In addition, T2-prolongation was observed in 6/14 drugs considered safe. This clearly indicates that the rat EHTs test does not sufficiently discriminate between high and low risk drugs, very likely due to the lack of $\mathrm{I}_{\mathrm{Kr}}$ sensitivity.

Nevertheless, screening the large number of drugs revealed a number of interesting novel informations. (1) It is apparent that $\mathrm{T} 2$ prolongations and beating irregularities were seen mainly in those drugs which reach high FTPC in clinical use and were therefore tested at high absolute concentrations (compare concentration range of Group 1 and 2 drugs with group 3 and 4; Fig. 8). This confirms the general rule in pharmacology that drugs with low potency at their target have a higher chance of off-target toxicity than high affinity drugs. (2) Most effects were seen at 30-100-fold FTPC and higher. This is at the upper limit generally considered a critical safety margin [31] and point to a relatively low sensitivity of our assay. Rather than indicating simple non-specific effects (drugs such as sematilide and ciprofloxacin were tested at up to $300 \mu \mathrm{M}$ ) the data with E-4031, HMR-1556 and 4AP indicate that almost complete block of one or more currents is required to overcome safety mechanisms of repolarization in a relatively intact system such as the EHT. As such, the low sensitivity probably reflects the situation in vivo better than isolated cells [28]. Pharmacokinetic peculiarities and timedependent effects likely add to high concentration requirements. For example, amiodarone is very lipophilic and accumulates in cells over time. With an apparent volume of distribution of $20-200 \mathrm{l} / \mathrm{kg}$, the low FTPC of amiodarone $(0.3 \mathrm{nM})$ is orders of magnitude lower than cellular concentrations in the steady state. (3) Concentrations in which group 1 and 2 drugs exerted their effects on EHTs were generally well above their $\mathrm{IC}_{50}$ for $\mathrm{hERG}$ (gray bars in Fig. 8). Notable exceptions are domperidone and phenytoin, where relaxation slowing occurred at concentrations below hERG $\mathrm{IC}_{50}$. Since E-4031 at high concentration markedly potentiated the effect of HMR-1556 without having an effect alone, the data suggest that inhibition of $\mathrm{I}_{\mathrm{Kr}}$ may participate in the effect of group 1 and 2 drugs, but is not sufficient to fully explain them. Inhibition of other currents must come into play. We found reports of $\mathrm{I}_{\mathrm{to}}$-inhibiting activity for 14 drugs (blue bars in Fig. 8). In four of these (imipramine, propafenone, quinidine, and clarithromycin) published $\mathrm{IC}_{50}$ values for $\mathrm{hERG}$ and $\mathrm{I}_{\mathrm{to}}$ were at or below the threshold for $\mathrm{T} 2$ prolongation or irregularity, providing a likely mechanism of action. Moreover, we found reports of $\mathrm{I}_{\mathrm{Ks}}$-inhibiting effects for 20 drugs (green bars in Fig. 8). In six of these cases (bepridil, thioridazine, propafenone, quinidine, cibenzoline, and sotalol), the $\mathrm{IC}_{50}$ was at or below the threshold for group 1 or 2 effects. It is interesting that quinidine at the T2threshold concentration $(100 \mu \mathrm{M})$ inhibits all three currents $\mathrm{I}_{\mathrm{Kr}}, \mathrm{I}_{\mathrm{to}}$, and $\mathrm{I}_{\mathrm{Ks}}$. Other currents involved in rat cardiac repolarization such as $\mathrm{I}_{\mathrm{K} 1}, \mathrm{I}_{\mathrm{ss}}$, and $\mathrm{I}_{\mathrm{Kx}}$ [19] or $\mathrm{I}_{\mathrm{KATP}}$ [4] and $\mathrm{I}_{\mathrm{KNa}}$ are also potential targets, but not much is known about effects of the drugs investigated. Taken together, the data indicate that many clinically used drugs, some of them associated with TdP, others not, inhibit cardiac repolarizing currents in addition to $\mathrm{I}_{\mathrm{Kr}}$. This supports recent data suggesting that combined channel block underlies clinical proarrhythmia [28].

\section{Miscellaneous effects on EHT function}

Some drugs caused T1 prolongations (haloperidol, sertindole, diphenhydramine, and mefloquine), T2 acceleration (terfenadine) or negative inotropic effects (verapamil). Whereas the latter is the expected main effect of the drug, the mechanism of the other is unclear at present. T1 prolongations could indicate reduced conduction velocity in the EHT, a typical consequence of $\mathrm{I}_{\mathrm{Na}}$ inhibition. However, pure $\mathrm{I}_{\mathrm{Na}}$ blockers such as TTX or lidocaine did not prolong T1 (data not shown), arguing against this idea. An alternative may be an effect on gap junction conduction. At least mefloquine is known to inhibit numerous connexins $(\mathrm{C} \times)$, including $\mathrm{C} \times 43$ [8]. T2 shortening could be due to accelerated repolarization by stimulation of K-currents or accelerated myofilament relaxation, but also due to an inhibition of $\mathrm{I}_{\mathrm{Na}}$. In fact, terfenadine, which also shortened action potential duration in the SCREENIT test system (10 $\mu \mathrm{M}$ [23]), has recently been suggested to cause 
arrhythmias not by a TdP mechanism, but by its strong $\mathrm{I}_{\mathrm{Na}^{-}}$ blocking activity [46].

Taken together, rat EHTs are not suitable as a general screening assay for proarrhythmic drug effects due to the small contribution of $\mathrm{I}_{\mathrm{Kr}}$ for rat EHT repolarization. On the other hand, the assay is a simple and robust system for the analysis of non-hERG-related drug effects on cardiac function. Relaxation time was shown to be a particularly suited screening parameter, sensitive to drugs affecting cardiac repolarization, but also $\mathrm{Ca}^{2+}$ handling or myofilament function. The high fraction of drugs with known arrhythmogenic effects that prolonged relaxation or induced irregular beating in this hERG-insensitive system suggest that $\mathrm{I}_{\text {to }}$ and $\mathrm{I}_{\mathrm{Ks}}$ effects add to the proarrhythmic risk of drugs and require further consideration.

Acknowledgments We are grateful for the technical help by Bülent Aksehirlioglu, Hamburg, in constructing the EHT setup and to Prof. Ursula Ravens, University of Dresden, for the kind gift of HMR-1556.

\section{Conflict of interest None.}

Funding sources This study was supported by grants from the German Research Foundation (DFG Es/88-12), the European Commission (FP7 Angioscaff and FP7 Biodesign) and the DZHK (German Centre for Cardiovascular Research), BMBF (German Ministry for Education and Research).

Open Access This article is distributed under the terms of the Creative Commons Attribution License which permits any use, distribution, and reproduction in any medium, provided the original author(s) and the source are credited.

\section{Appendix}

EHTs were adopted from Redfern et al. 2003. Case reports (indicated by asterisk), estimated plasma concentration and IC50 values on Ito/IKs were obtained by a literature search.

${ }^{1}$ William S Redfern et al. 2003; Cardiovasc Res

${ }^{2}$ Daniel Scagliotti et al. 1982; Am J Cardiol

${ }^{3}$ Maasaki Yasuda et al. 2006; Circ J

${ }^{4}$ Martin Karch et al. 1997; Herz

${ }^{5}$ Toshihiro Ohata et al. 2010; Thorac Surg

${ }^{6}$ Joao V Vitola et al. 1998; J Cardiovasc Electrophysiol

${ }^{7}$ Hideyuki Kamochi et al. 1999; Jpn Circ J

${ }^{8}$ Franco Casazza et al. 1986; G Ital Cardiol

${ }^{9}$ Dagfinn AArskog and Asmund Reikvam 1992; Tidsskr Nor Laegefore

${ }^{10}$ Etienne Delacrétaz and Jürg Fuhrer 1999; Clin Cardiol

${ }^{11}$ Charlotte van Noord et al. 2010; Drug Saf

${ }^{12}$ Arthur J Moss and Joel Morganroth 1999; Drug Saf

${ }^{13}$ Michael W Brandriss et al. 1994; Clin Infect Dis

${ }^{14}$ Yigal Pinto et al. 1999; Lancet

${ }^{15}$ Juan M Nogales Asensio et al. 2007; Int J Cardiol

${ }^{16} \mathrm{KM}$ Goel and RA Shanks; $\mathrm{Br}$ Med J
${ }^{17}$ Ngai-Shing Mok et al. 2005; J Cardiovasc Electrophysiol

${ }^{18}$ Winoc Fonteyne et al. 1996; Clin Cardiol

${ }^{19}$ John C DeToledo et al. 2001; Epilepsia

${ }^{20}$ Dan Tzivoni et al. 1981; Arch Intern Med

${ }^{21}$ John T Hii et al. 1991; Pacing Clin Electrophysiol

${ }^{22}$ Kwadwo Amankwa 2004; Clin Pharmacol Ther

${ }^{23}$ Wladyslaw Sinkiewicz 2006; Pol Arch Med Wewn

${ }^{24}$ Quian-Yong Liu et al. 1997; Acta Pharmacol Sin

${ }^{25}$ Ki-Wug Sung 2013; Naunyn Schmiedebers Arch P

${ }^{26}$ Dominique Abela 2010; Birth Defects Res B Dev

${ }^{27}$ Dong Zhang et al. 2011; Acta Pharmacol Sin

${ }^{28}$ Sean A Cahill et al. 2001; J Cardiovasc Pharmacol

${ }^{29}$ Pascale Gluais et al. 2003; Fundam Clin Pharmacol

${ }^{30}$ F Berger et al. 1989; Naunyn Schmiedebers Arch P

${ }^{31} \mathrm{~L} \mathrm{Xu}$ et al. 2008; Pharmazie

${ }^{32}$ Pascale Gluais et al. 2004; Eur J Pharmacol

${ }^{33}$ Oscar Casis et al. 1998; J Cardiovasc Pharmacol

${ }^{34}$ Imju Jeong et al. 2013; Brain Res

${ }^{35}$ Aaref Badshah et al. 2009; Am J Med Sci

${ }^{36}$ Yoshihiro Yumoto et al. 2004; J Cardiovasc Pharmacol

${ }^{37}$ Benoit Drolet et al. 1999; J Pharmacol Exp Ther

${ }^{38}$ Dao Wu Wang et al. 1996; J Moll Cell Cardiol

${ }^{39}$ Ling-Ping Lai et al. 1999; J Biomed Sci

${ }^{40}$ Joseph J Salata et al. 1995; Circ Res

${ }^{41}$ Jisheng Kang et al. 2001; J Pharmacol Exp Ther

${ }^{42}$ Jisheng Kang et al. 2000; Eur J Pharmacol

${ }^{43}$ Stephen E Jones et al. 1998; Br J Pharmacol

${ }^{44}$ Christiaan C Veerman et al. 2013; Circ Arrhythm Electrophysiol

${ }^{45}$ Edward Carmeliet 1998; Br J Pharmacol

\section{References}

1. Ackerman MJ, Mohler PJ (2010) Defining a new paradigm for human arrhythmia syndromes: phenotypic manifestations of gene mutations in ion channel- and transporter-associated proteins. Circ Res 107:457-465. doi:10.1161/CIRCRESAHA.110.224592

2. Braam SR, Tertoolen L, van de Stolpe A, Meyer T, Passier R, Mummery CL (2010) Prediction of drug-induced cardiotoxicity using human embryonic stem cell-derived cardiomyocytes. Stem Cell Res 4:107-116. doi:10.1016/j.scr.2009.11.004

3. Brown AM (2009) High throughput functional screening of an ion channel library for drug safety and efficacy. Eur Biophys $\mathbf{J}$ 38:273-278. doi:10.1007/s00249-008-0356-2

4. Burley DS, Cox CD, Zhang J, Wann KT, Baxter GF (2014) Natriuretic peptides modulate ATP-sensitive $\mathrm{K}(+)$ channels in rat ventricular cardiomyocytes. Basic Res Cardiol 109:402. doi:10. 1007/s00395-014-0402-4

5. Cerignoli F, Charlot $D$, Whittaker R, Ingermanson R, Gehalot $P$, Savchenko A, Gallacher DJ, Towart R, Price JH, McDonough PM, Mercola M (2012) High throughput measurement of $\mathrm{Ca}^{2+}$ dynamics for drug risk assessment in human stem cell-derived cardiomyocytes by kinetic image cytometry. J Pharmacol Toxicol Methods 66:246-256. doi:10.1016/j.vascn.2012.08.167 
6. Christ T, Galindo-Tovar A, Thoms M, Ravens U, Kaumann AJ (2009) Inotropy and L-type $\mathrm{Ca}_{2}{ }^{+}$current, activated by beta1- and beta2-adrenoceptors, are differently controlled by phosphodiesterases 3 and 4 in rat heart. Br J Pharmacol 156:62-83. doi:10. 1111/j.1476-5381.2008.00015.x

7. Crocini C, Arimura T, Reischmann S, Eder A, Braren I, Hansen A, Eschenhagen T, Kimura A, Carrier L (2013) Impact of ANKRD1 mutations associated with hypertrophic cardiomyopathy on contraction parameters of engineered heart tissue. Basic Res Cardiol 108:349. doi:10.1007/s00395-013-0349-x

8. Cruikshank SJ, Hopperstad M, Younger M, Connors BW, Spray DC, Srinivas M (2004) Potent block of $C \times 36$ and $C \times 50$ gap junction channels by mefloquine. Proc Natl Acad Sci USA 101:12364-12369. doi:10.1073/pnas.0402044101

9. Davis J, Wen H, Edwards T, Metzger JM (2008) Allele and species dependent contractile defects by restrictive and hypertrophic cardiomyopathy-linked troponin I mutants. J Mol Cell Cardiol 44:891-904. doi:10.1016/j.yjmcc.2008.02.274

10. Echt DS, Liebson PR, Mitchell LB, Peters RW, Obias-Manno D, Barker AH, Arensberg D, Baker A, Friedman L, Greene HL (1991) Mortality and morbidity in patients receiving encainide, flecainide, or placebo. The cardiac arrhythmia suppression trial. N Engl J Med 324:781-788. doi:10.1056/NEJM199103213241201

11. EMA (2005) The nonclinical evaluation of the potential for delayed ventricular repolarization ( $Q t$ interval prolongation) by human pharmaceuticals S7B

12. FDA (2005) Guidance for industry S7B nonclinical evaluation of the potential for delayed ventricular repolarization (QT interval prolongation) by human pharmaceuticals

13. Fossa AA, Depasquale MJ, Raunig DL, Avery MJ, Leishman DJ (2002) The relationship of clinical QT prolongation to outcome in the conscious dog using a beat-to-beat QT-RR interval assessment. Pharmacology 202:828-833. doi:10.1124/jpet.102.035220

14. Gintant GA (2008) Preclinical Torsades-de-Pointes screens: advantages and limitations of surrogate and direct approaches in evaluating proarrhythmic risk. Pharmacol Ther 119:199-209. doi:10.1016/j.pharmthera.2008.04.010

15. Gintant GA, Limberis JT, McDermott JS, Wegner CD, Cox BF (2001) The canine Purkinje fiber: an in vitro model system for acquired long QT syndrome and drug-induced arrhythmogenesis. J Cardiovasc Pharmacol 37:607-618

16. Hansen A, Eder A, Bönstrup M, Flato M, Mewe M, Schaaf S, Aksehirlioglu B, Schwoerer AP, Schwörer A, Uebeler J, Eschenhagen T (2010) Development of a drug screening platform based on engineered heart tissue. Circ Res 107:35-44. doi:10. 1161/CIRCRESAHA.109.211458

17. Haverkamp W, Breithardt G, Camm aJ, Janse MJ, Rosen MR, Antzelevitch C, Escande D, Franz M, Malik M, Moss a, Shah R (2000) The potential for QT prolongation and pro-arrhythmia by non-anti-arrhythmic drugs: clinical and regulatory implications. Report on a Policy Conference of the European Society of Cardiology. Cardiovasc Res 47:219-233. doi:10.1016/S00086363(00)00119-X

18. Hilliard FA, Steele DS, Laver D, Yang Z, Le SJ, Chopra N, Piston DW, Huke S, Knollmann BC (2010) Flecainide inhibits arrhythmogenic $\mathrm{Ca}^{2+}$ waves by open state block of ryanodine receptor $\mathrm{Ca}^{2+}$ release channels and reduction of $\mathrm{Ca}^{2+}$ spark mass. J Mol Cell Cardiol 48:293-301. doi:10.1016/j.yjmcc.2009.10.005

19. Himmel HM, Wettwer E, Li Q, Ravens U (1999) Four different components contribute to outward current in rat ventricular myocytes Four different components contribute to outward current in rat ventricular myocytes. Am J Physiol 277:H107-H118

20. Hirt MN, Sörensen NA, Bartholdt LM, Boeddinghaus J, Schaaf S, Eder A, Vollert I, Stöhr A, Schulze T, Witten A, Stoll M, Hansen $A$, Eschenhagen $T$ (2012) Increased afterload induces pathological cardiac hypertrophy: a new in vitro model. Basic Res Cardiol 107:307. doi:10.1007/s00395-012-0307-z

21. Hoffmann P, Warner B (2006) Are hERG channel inhibition and QT interval prolongation all there is in drug-induced torsadogenesis? A review of emerging trends. J Pharmacol Toxicol Methods 53:87-105. doi:10.1016/j.vascn.2005.07.003

22. Hondeghem LM, Carlsson L, Duker G (2001) Instability and triangulation of the action potential predict serious proarrhythmia, but action potential duration prolongation is antiarrhythmic. Circulation 103:2004-2013. doi:10.1161/01.CIR.103.15.2004

23. Hondeghem LM, Dujardin K, Hoffmann P, Case IA, Of S (2011) Drug-induced QT C prolongation dangerously underestimates proarrhythmic potential : lessons from terfenadine. Baseline 57:589-597. doi:10.1097/FJC.0b013e3182135e91

24. Hondeghem LM, Hoffmann P (2003) Blinded test in isolated female rabbit heart reliably identifies action potential duration prolongation and proarrhythmic drugs: importance of triangulation, reverse use dependence, and instability. J Cardiovasc Pharmacol 41:14-24

25. Laverty H, Benson C, Cartwright E, Cross M, Garland C, Hammond T, Holloway C, McMahon N, Milligan J, Park B, Pirmohamed M, Pollard C, Radford J, Roome N, Sager P, Singh S, Suter T, Suter W, Trafford a, Volders P, Wallis R, Weaver R, York M, Valentin J (2011) How can we improve our understanding of cardiovascular safety liabilities to develop safer medicines? Br J Pharmacol 163:675-693. doi:10.1111/j.14765381.2011.01255.x

26. Lipinski CA, Lombardo F, Dominy BW, Feeney PJ (2001) Experimental and computational approaches to estimate solubility and permeability in drug discovery and development settings. Adv Drug Deliv Rev 46:3-26. doi:10.1016/S0169-409X(00)00 129-0

27. Milan DJ, Peterson TA, Ruskin JN, Peterson RT, Calum A (2003) Drugs that induce repolarization abnormalities cause bradycardia in zebrafish. Circulation 107:1355-1358. doi:10.1161/01.CIR. 0000061912.88753 .87

28. Nalos L, Varkevisser R, Jonsson MKB, Houtman MJC, Beekman JD, van der Nagel R, Thomsen MB, Duker G, Sartipy P, de Boer TP, Peschar M, Rook MB, van Veen TAB, van der Heyden MAG, Vos MA (2012) Comparison of the IKr blockers moxifloxacin, dofetilide and E-4031 in five screening models of proarrhythmia reveals lack of specificity of isolated cardiomyocytes. Br J Pharmacol 165:467-478. doi:10.1111/j.1476-5381.2011. 01558.x

29. Nerbonne JM, Kass RS (2005) Molecular physiology of cardiac repolarization. Physiol Rev 85:1205-1253. doi:10.1152/physrev. 00002.2005

30. Qian J-Y, Guo L (2010) Altered cytosolic $\mathrm{Ca}^{2+}$ dynamics in cultured Guinea pig cardiomyocytes as an in vitro model to identify potential cardiotoxicants. Toxicol In Vitro 24:960-972. doi:10.1016/j.tiv.2009.12.027

31. Redfern WS, Carlsson L, Davis aS, Lynch WG, MacKenzie I, Palethorpe S, Siegl PKS, Strang I, Sullivan aT, Wallis R, Camm aJ, Hammond TG (2003) Relationships between preclinical cardiac electrophysiology, clinical QT interval prolongation and torsade de pointes for a broad range of drugs: evidence for a provisional safety margin in drug development. Cardiovasc Res 58:32-45. doi:10.1016/S0008-6363(02)00846-5

32. Regan CP, Cresswell HK, Zhang R, Lynch JJ (2005) Novel method to assess cardiac electrophysiology in the rat: characterization of standard ion channel blockers. J Cardiovasc Pharmacol 46:68-75. doi:10.1097/01.fjc.0000162774.86780.9d

33. Schaaf S, Shibamiya A, Mewe M, Eder A, Stöhr A, Hirt MN, Rau T, Zimmermann W-H, Conradi L, Eschenhagen T, Hansen A (2011) Human engineered heart tissue as a versatile tool in basic 
research and preclinical toxicology. PLoS One 6:e26397. doi:10. 1371/journal.pone.0026397

34. Solaro RJ, Gambassi G, Warshaw DM, Keller MR, Spurgeon Ha, Spurgeon Ha, Beier N, Lakatta EG (1993) Stereoselective actions of thiadiazinones on canine cardiac myocytes and myofilaments. Circ Res 73:981-990. doi:10.1161/01.RES.73.6.98135

35. Braam SR, Tertoolen L, van de Stolpe A, Meyer T, Passier R, Mummery CL (2010) Prediction of drug-induced cardiotoxicity using human embryonic stem cell-derived cardiomyocytes. Stem Cell Res 4:107-116. doi:10.1016/j.scr.2009.11.004

36. Stöhr A, Friedrich FW, Flenner F, Geertz B, Eder A, Schaaf S, Hirt MN, Uebeler J, Schlossarek S, Carrier L, Hansen A, Eschenhagen T (2013) Contractile abnormalities and altered drug response in engineered heart tissue from Mybpc3-targeted knockin mice. J Mol Cell Cardiol 63:189-198. doi:10.1016/j.yjmcc. 2013.07.011

37. Thomas GP, Gerlach U, Antzelevitch C (2003) HMR 1556, a potent and selective blocker of slowly activating delayed rectifier potassium current. J Cardiovasc Pharmacol 41:140-147

38. Thomsen MB, Matz J, Volders PGa, Vos MA (2006) Assessing the proarrhythmic potential of drugs: current status of models and surrogate parameters of torsades de pointes arrhythmias. Pharmacol Ther 112:150-170. doi:10.1016/j.pharmthera.2005.04.009

39. Usdin S, Haan K (2003) Chapter 11. In: The QT mandate, BioCentury, Washington, pp A1-A5

40. Vandenberg JI, Walker BD, Campbell TJ (2001) HERG K+ channels: friend and foe. Trends Pharmacol Sci 22:240-246. doi:10.1016/S0165-6147(00)01662-X

41. Venetucci LA, Trafford AW, O'Neill SC, Eisner DA (2007) Na/ $\mathrm{Ca}$ exchange: regulator of intracellular calcium and source of arrhythmias in the heart. Ann N Y Acad Sci 1099:315-325. doi:10.1196/annals.1387.033

42. Volk T, Nguyen TH, Schultz JH, Ehmke H (1999) Relationship between transient outward $\mathrm{K}+$ current and $\mathrm{Ca}^{2+}$ influx in rat cardiac myocytes of endo- and epicardial origin. J Physiol 519(3):841-850. doi:10.1111/j.1469-7793.1999.0841n.x

43. Wible BA, Kuryshev YA, Smith SS, Liu Z, Brown AM (2008) An ion channel library for drug discovery and safety screening on automated platforms. Assay Drug Dev Technol 6:765-780. doi:10.1089/adt.2008.0171

44. Zhou Z, Gong Q, Ye B, Fan Z, Makielski JC, Robertson GA, January CT (1998) Properties of HERG channels stably expressed in HEK 293 cells studied at physiological temperature. Biophys J 74:230-241

45. Zimmermann WH, Fink C, Kralisch D, Remmers U, Weil J, Eschenhagen T (2000) Three-dimensional engineered heart tissue from neonatal rat cardiac myocytes. Biotechnol Bioeng 68:106-114. doi:10.1002/(SICI)1097-0290(20000405)6

46. Zimmermann W-H, Schneiderbanger K, Schubert P, Didié M, Münzel F, Heubach JF, Kostin S, Neuhuber WL, Eschenhagen T (2002) Tissue engineering of a differentiated cardiac muscle construct. Circ Res 90:223-230. doi:10.1161/hh0202.103644

47. Lu HR, Hermans AN, Gallacher DJ (2012) Does terfenadineinduced ventricular tachycardia/fibrillation directly relate to its QT prolongation and Torsades de Pointes? $\mathrm{Br} \mathrm{J}$ Pharmacol 166:1490-1520. doi:10.1111/j.1476-5381.2012.01880.x 